\title{
Investigation of Free Particle Propagator with Generalized Uncertainty Problem
}

\author{
F. Ghobakhloo and H. Hassanabadi \\ Physics Department, Shahrood University of Technology, P.O. Box 316, Shahrood 3619995161, Iran \\ Correspondence should be addressed to F. Ghobakhloo; fatemeh.ghobakhloo@gmail.com
}

Received 1 March 2016; Revised 11 April 2016; Accepted 24 April 2016

Academic Editor: Mir Faizal

Copyright ( 2016 F. Ghobakhloo and H. Hassanabadi. This is an open access article distributed under the Creative Commons Attribution License, which permits unrestricted use, distribution, and reproduction in any medium, provided the original work is properly cited. The publication of this article was funded by SCOAP ${ }^{3}$.

\begin{abstract}
We consider the Schrödinger equation with a generalized uncertainty principle for a free particle. We then transform the problem into a second-order ordinary differential equation and thereby obtain the corresponding propagator. The result of ordinary quantum mechanics is recovered for vanishing minimal length parameter.
\end{abstract}

\section{Introduction}

The generalization of classical action principle into quantum theory appears in path integral formulation. Instead of a single classical path, the quantum version considers a sum, or better, say, integral, of infinite possible paths [1,2]. Although the main idea of path integral approach was released by $\mathrm{N}$. Wiener, in an attempt to solve diffusion and Brownian problems, it was introduced in Lagrangian formulation of quantum mechanics of P. A. M. Dirac [1, 2]. Nevertheless, the present comprehensive formulation is named after Feynman and extracted from his Ph.D. thesis supervised by J. A. Wheeler [1, 2]. Feynman's formulation is now an essential ingredient in many fundamental theories of theoretical physics including quantum field theory, quantum gravity, and high energy physics [1-3].

On the other hand, we are now almost sure from fundamental theories such as string theory and quantum gravity that the ordinary quantum mechanics ought to be reformulated. In more precise words, a generalization of Heisenberg uncertainty principle, called generalized uncertainty principle (GUP), should be considered at energies of order Planck scale [4-7]. This generalization corresponds to a generalization of wave equation of quantum mechanics. Till now, various wave equations of quantum mechanics, different interactions, and other related mathematical aspects and physical concepts have been considered in this framework [8-18].
In our paper, we are going to combine these two subjects. Namely, we study the free particle propagator in Schrödinger framework in minimal length formulation. In Section 2, we review the essential concepts of GUP and write the generalized Hamiltonian for free particle. In Section 3, we obtain the propagator for this system in which the details of calculations are brought.

\section{GUP-Corrected Hamiltonian}

An immediate consequence of the ML is the GUP

$$
\Delta x \geq \frac{\hbar}{\Delta p}+\alpha l_{p}^{2} \frac{\Delta p}{\hbar},
$$

where the GUP parameter $\alpha$ is determined from a fundamental theory. At low energies, that is, energies much smaller than the Planck mass, the second term on the right hand side of (1) vanishes and we recover the well-known Heisenberg uncertainty principle. The GUP of (1) corresponds to the generalized commutation relation

$$
\left[x_{o p}, p_{o p}\right]=i \hbar\left(1+\beta p^{2}\right) \quad 0 \leq \beta \leq 1,
$$

where $x_{o p}=x, p_{o p}=p\left[1+\beta(p)^{2}\right]$ and $0 \leq \beta \leq 1$. The limits $\beta \rightarrow 0$ and $\beta \rightarrow 1$ correspond to the standard quantum mechanics and extreme quantum gravity, respectively. 
Equation (2) gives the minimal length in this case as $(\Delta x)_{\min }=2 l_{p} \sqrt{\alpha}$. It should be noted that, in the deformed Schrödinger equation, the Hamiltonian does not have any explicit time dependence [19]:

$$
\left(\frac{p_{o p}^{2}}{2 m}+V(x)\right) \psi_{n}(x)=E_{n} \psi_{n}(x)
$$

This deformed momentum operator modifies the original Hamiltonian as

$$
H=\frac{p^{2}}{2 m}+V_{f f}(x)
$$

where

$$
V_{f f}(x)=\beta \frac{p^{4}}{m}+V(x) .
$$

The problem becomes much simpler if we consider [16]

$$
\begin{aligned}
& p^{2}=2 m\left(E_{n}^{(0)}-V(x)\right), \\
& p^{4}=4 m^{2}\left(E_{n}^{(0)}-V(x)\right)^{2} .
\end{aligned}
$$

In the free particle case, we therefore have

$$
H=\frac{p^{2}}{2 m}+4 \beta m\left(E_{n}^{(0)}\right)^{2}
$$

We now calculate the single free particle propagator corresponding to this deformed Hamiltonian in Section 3.

\section{Perspicuous Form of Propagator}

If the wave function $\psi\left(x, t^{\prime}\right)$ is known at a time $t^{\prime}$, we can explicitly write the wave function $\psi\left(x, t^{\prime \prime}\right)$ at a later time $t^{\prime \prime}$ using the propagation relation as [20]

$$
\psi\left(x, t^{\prime \prime}\right)=\exp \left(-\frac{i H\left(t^{\prime \prime}-t^{\prime}\right)}{\hbar}\right) \psi\left(x, t^{\prime}\right) .
$$

For a small time interval $t^{\prime \prime}-t^{\prime}=\Delta t$, we have

$$
\begin{aligned}
& \left\langle x\left|\exp \left(-\frac{i H \Delta t}{\hbar}\right)\right| x\right\rangle=\frac{1}{2 \pi \hbar} \int d p \\
& \cdot\left(\left\langle x\left|\exp -\frac{i}{\hbar}\left(\frac{p^{2}}{2 m} \Delta t\right)\right| p\right\rangle\right. \\
& \left.\cdot\left\langle p\left|\exp -\frac{i}{\hbar}\left(4 \beta m\left(E_{n}^{(0)}\right)^{2} \Delta t\right)\right| x\right\rangle\right)=\int \frac{d p}{2 \pi \hbar} \\
& \cdot \exp -\frac{i}{\hbar}\left(\frac{p^{2}}{2 m}+4 \beta m\left(E_{n}^{(0)}\right)^{2} \Delta t\right) .
\end{aligned}
$$

Therefore, the quantum mechanical propagator for small time interval $\Delta t=t-t^{\prime}$, corresponding to this nonlocal Hamiltonian, can be written as

$$
K\left(x^{\prime \prime}, t^{\prime \prime} ; x^{\prime}, t^{\prime}\right)=\int \exp \left(\frac{i}{\hbar} \int_{t^{\prime}}^{t^{\prime}+\Delta t} L(t) d t\right) \frac{d p}{2 \pi \hbar},
$$

in which the Lagrangian is given by [20]

$$
L(t)=p \cdot \frac{\left(x^{\prime \prime}-x^{\prime}\right)}{\left(t^{\prime \prime}-t^{\prime}\right)}-\frac{p^{2}}{2 m}-4 \beta m\left(E_{n}^{(0)}\right)^{2} .
$$

Therefore, the propagator appears as

$$
\begin{aligned}
& K\left(x^{\prime \prime}, t^{\prime \prime} ; x^{\prime}, t^{\prime}\right)=\frac{1}{2 \pi \hbar} \\
& \cdot \exp \frac{i}{\hbar}\left(\frac{\left(x^{\prime \prime}-x^{\prime}\right)^{2} m}{2 \Delta t}-4 \beta m\left(E_{n}^{(0)}\right)^{2} \Delta t\right) \\
& \cdot \int d p \cdot \exp \left(-\frac{i \Delta t}{2 m \hbar}\left(p-\frac{\left(x^{\prime \prime}-x^{\prime}\right) m}{\Delta t}\right)^{2}\right)
\end{aligned}
$$

or

$$
\begin{aligned}
& K\left(x^{\prime \prime}, t^{\prime \prime} ; x^{\prime}, t^{\prime}\right)=\frac{1}{2 \pi \hbar} \\
& \cdot \exp \frac{i}{\hbar}\left(\frac{\left(x^{\prime \prime}-x^{\prime}\right)^{2} m}{2 \Delta t}-4 \beta m\left(E_{n}^{(0)}\right)^{2} \Delta t\right) \int d U \\
& \cdot \exp \left(-\frac{i \Delta t}{2 m \hbar} U^{2}\right)
\end{aligned}
$$

with

$$
U=\left(p-\frac{\left(x^{\prime \prime}-x^{\prime}\right) m}{\Delta t}\right)
$$

In a more explicit form, the propagator for free particle under minimal length is

$$
\begin{aligned}
& K\left(x^{\prime \prime}, t^{\prime \prime} ; x^{\prime}, t^{\prime}\right)=\left(\sqrt{\frac{m}{2 i \pi \hbar \Delta t}}\right) \\
& \quad \cdot \exp \frac{i}{\hbar}\left(\frac{\left(x^{\prime \prime}-x^{\prime}\right)^{2} m}{2\left(t^{\prime \prime}-t^{\prime}\right)}-4 \beta m\left(E_{n}^{(0)}\right)^{2}\left(t^{\prime \prime}-t^{\prime}\right)\right) .
\end{aligned}
$$

Now, if we assume $\beta=0$, then the one-dimensional free particle propagator is given by

$$
\begin{aligned}
& K\left(x^{\prime \prime}, t^{\prime \prime} ; x^{\prime}, t^{\prime}\right) \\
& \quad=\left(\sqrt{\frac{m}{2 i \pi \hbar \Delta t}}\right) \exp \frac{i}{\hbar}\left(\frac{\left(x^{\prime \prime}-x^{\prime}\right)^{2} m}{2\left(t^{\prime \prime}-t^{\prime}\right)}\right) .
\end{aligned}
$$

In order to obtain free particle propagator for a finite time interval $\left(t^{\prime \prime}-t^{\prime}\right)$ we divide the interval into $N$ subintervals of 
equal length $\Delta t$ such that $\left(t^{\prime \prime}-t^{\prime}\right)=N \Delta t$. Now, the propagator of a finite time interval is written as

$$
\begin{aligned}
& K\left(x^{\prime \prime}, t^{\prime \prime} ; x^{\prime}, t^{\prime}\right)=\left(\sqrt{\frac{m}{2 i \pi \hbar \Delta t}}\right)^{N} \\
& \cdot \int d x_{1} d x_{2} d x_{3} \cdots d x_{N-1} \exp \frac{i m}{2 \hbar \Delta t} \\
& \cdot\left(\left(x_{1}-x_{0}\right)^{2}+\left(x_{2}-x_{1}\right)^{2}+\cdots+\left(x_{N}-x_{N-1}\right)^{2}\right) \\
& \cdot \exp \left(\frac{-i 4 \beta m}{\hbar}\left(E_{n}^{(0)}\right)^{2}\left(t^{\prime \prime}-t^{\prime}\right)\right) .
\end{aligned}
$$

The integral in (17) can be calculated as [20]

$$
\begin{aligned}
& \int d x_{1} d x_{2} d x_{3} \cdots d x_{N-1} \exp i \lambda \\
& \cdot\left(\left(x_{1}-x_{0}\right)^{2}+\left(x_{2}-x_{1}\right)^{2}+\cdots+\left(x_{N}-x_{N-1}\right)^{2}\right) \\
& =\frac{1}{\sqrt{N}}\left(\frac{i \pi}{\lambda}\right)^{(N-1) / 2} \exp \left(\frac{i \lambda\left(x_{N}-x_{0}\right)^{2}}{N}\right) .
\end{aligned}
$$

Substituting (18) into (17), the propagator is obtained as

$$
\begin{aligned}
K\left(x^{\prime \prime}, t^{\prime \prime} ; x^{\prime}, t^{\prime}\right)= & \left(\sqrt{\frac{m}{2 i \pi \hbar \Delta t}}\right)^{N} \frac{1}{\sqrt{N}}\left(\frac{i \pi}{\lambda}\right)^{(N-1) / 2} \\
& \cdot \exp \left(\frac{i \lambda\left(x_{N}-x_{0}\right)^{2}}{N}\right) \\
& \cdot \exp \left(\frac{-i 4 \beta m}{\hbar}\left(E_{n}^{(0)}\right)^{2} \Delta t\right)
\end{aligned}
$$

where $\lambda=m / 2 \hbar \Delta t$. Replacing $x_{N}$ and $x_{0}$ by $x^{\prime \prime}$ and $x^{\prime}$, respectively, and using $\left(t^{\prime \prime}-t^{\prime}\right)=N \Delta t$, we obtain the final expression as

$$
\begin{aligned}
K\left(x^{\prime \prime}, t^{\prime \prime} ; x^{\prime}, t^{\prime}\right)= & \left(\sqrt{\frac{m}{2 i \pi \hbar \Delta t}}\right)^{N} \frac{1}{\sqrt{N}}\left(\frac{\lambda}{i \pi}\right)^{(N-1) / 2} \\
& \cdot \exp \left(\frac{i \lambda\left(x_{N}-x_{0}\right)^{2}}{N}\right) \\
& \cdot \exp \left(\frac{-i 4 \beta m}{\hbar}\left(E_{n}^{(0)}\right)^{2} \Delta t\right)
\end{aligned}
$$

Now, if we calculate the probability of detecting the particle at a finite region $\Delta x$, enclosing final point $x^{\prime \prime}$, from (20), we get

$$
\begin{aligned}
K\left(x^{\prime \prime}, t^{\prime \prime} ; x^{\prime}, t^{\prime}\right)= & \left(\sqrt{\frac{m}{2 i \pi N \hbar \Delta t}}\right) \\
& \cdot \exp \left(\frac{i m\left(x_{N}-x_{0}\right)^{2}}{2 \hbar N \Delta t}\right) \\
& \cdot \exp \left(\frac{i 4 \beta m}{\hbar}\left(E_{n}^{(0)}\right)^{2} \Delta t\right) .
\end{aligned}
$$

In the limit $\beta \rightarrow 0$, the final form of propagator is given by

$$
\begin{aligned}
K & \left(x^{\prime \prime}, t^{\prime \prime} ; x^{\prime}, t^{\prime}\right) \\
& =\left(\sqrt{\frac{m}{2 i \pi \hbar\left(t^{\prime \prime}-t^{\prime}\right)}}\right) \exp \left(\frac{i m\left(x^{\prime \prime}-x^{\prime}\right)^{2}}{2 \hbar\left(t^{\prime \prime}-t^{\prime}\right)}\right)
\end{aligned}
$$

which is the result in ordinary quantum mechanics.

\section{Conclusion}

We considered the nonrelativistic free particle propagation problem in an analytical manner in minimal length formalism. We first transformed arising differential equation into a second-order differential equation which included a modified effective potential. We next calculated the propagator. Apart from the application of the study, the work is of pedagogical interest in graduate physics.

\section{Competing Interests}

The authors declare that they have no competing interests.

\section{References}

[1] M. Chaichian and A. Demichev, Path Integrals in Physics, Institute of Physics, Bristol, UK, 2001.

[2] R. P. Feynman and A. R. Hibbs, Quantum Mechanics and Path Integrals, McGrawhill, New York, NY, USA, 1965.

[3] J. J. Sakurai, Modern Quantum Mechanics, Addison-Wesley, Reading, Mass, USA, 1994.

[4] A. F. Ali, M. M. Khalil, and E. C. Vagenas, "Minimal length in quantum gravity and gravitational measurements," Europhysics Letters, vol. 112, no. 2, Article ID 20005, 2015.

[5] S. Das, E. C. Vagenas, and A. F. Ali, "Discreteness of space from GUP II: relativistic wave equations," Physics Letters B, vol. 690, no. 4, pp. 407-412, 2010.

[6] W. Chemissany, S. Das, A. F. Ali, and E. C. Vagenasc, "Effect of the generalized uncertainty principle on post-inflation preheating," Journal of Cosmology and Astroparticle Physics, vol. 12, article 017, 2011.

[7] A. Kempf and G. Mangano, "Minimal length uncertainty relation and ultraviolet regularization," Physical Review D, vol. 55, no. 12, pp. 7909-7920, 1997.

[8] L. J. Garay, "Quantum gravity and minimum length," International Journal of Modern Physics A, vol. 10, no. 2, pp. 145-166, 1995.

[9] A. N. Tawfik and A. M. Diab, "Review on generalized uncertainty principle," http://arxiv.org/abs/1509.02436.

[10] S. Gangopadhyay, A. Dutta, and M. Faizal, "Constraints on the generalized uncertainty principle from black-hole thermodynamics," Europhysics Letters, vol. 112, no. 2, Article ID 20006, 2015.

[11] H. Hassanabadi, S. Zarrinkamar, and E. Maghsoodi, "Scattering states of Woods-Saxon interaction in minimal length quantum mechanics," Physics Letters B, vol. 718, no. 2, pp. 678-682, 2012.

[12] S. Das and E. C. Vagenas, "Universality of quantum gravity corrections," Physical Review Letters, vol. 101, no. 22, Article ID 221301, 2008 
[13] K. Jahankohan, S. Zarrinkamar, and H. Hassanabadi, "Cornell potential in generalized uncertainty principle formalism: the case of Schrödinger equation," Quantum Studies: Mathematics and Foundations, vol. 3, no. 1, pp. 109-114, 2016.

[14] K. Jahankohan, H. Hassanabadi, and S. Zarrinkamar, "Relativistic Ramsauer-Townsend effect in minimal length framework," Modern Physics Letters A, vol. 30, no. 32, Article ID 1550173, 8 pages, 2015.

[15] S. Haouat, "Schrödinger equation and resonant scattering in the presence of a minimal length," Physics Letters B, vol. 729, pp. 3338, 2014.

[16] D. Kothawala, S. Shankaranarayanan, and L. Sriramkumar, "Quantum gravitational corrections to the propagator in spacetimes with constant curvature," http://arxiv.org/abs/1002.1132.

[17] F. de Felice, M. T. Crosta, A. Vecchiato, M. G. Lattanzi, and B. Bucciarelli, "A general relativistic model of light propagation in the gravitational field of the solar system: the static case," Astrophysical Journal, vol. 607, no. 1 I, pp. 580-595, 2004.

[18] A. Maas, "Some more details of minimal-Landau-gauge SU(2) Yang-Mills propagators," Physical Review D, vol. 91, Article ID 034502, 2015.

[19] H. Hassanabadi, S. Zarrinkamar, and E. Maghsoodi, "Scattering states of Woods-Saxon interaction in minimal length quantum mechanics," Physics Letters B, vol. 718, no. 2, pp. 678-682, 2012.

[20] S. Pramanik, M. Faizal, M. Moussa, and A. Farag Ali, "Path integral quantization corresponding to the deformed Heisenberg algebra," Annals of Physics, vol. 362, pp. 24-35, 2015. 

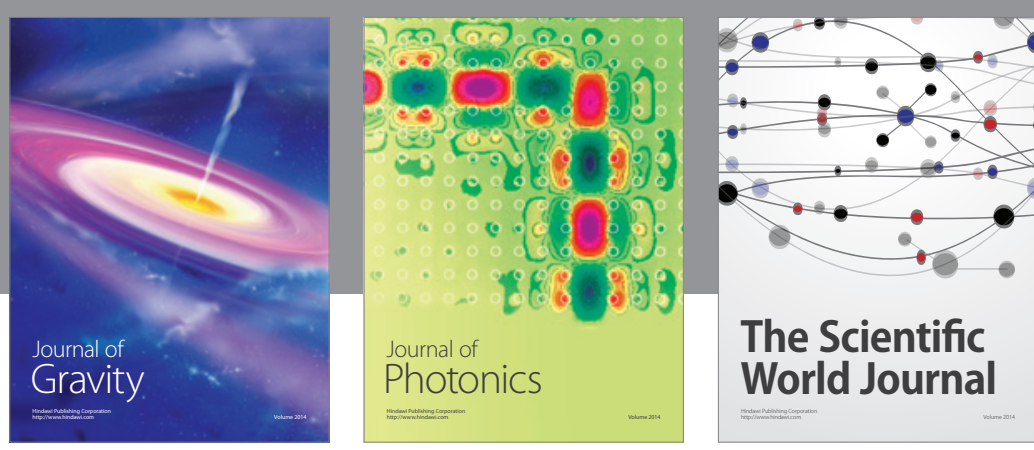

The Scientific World Journal
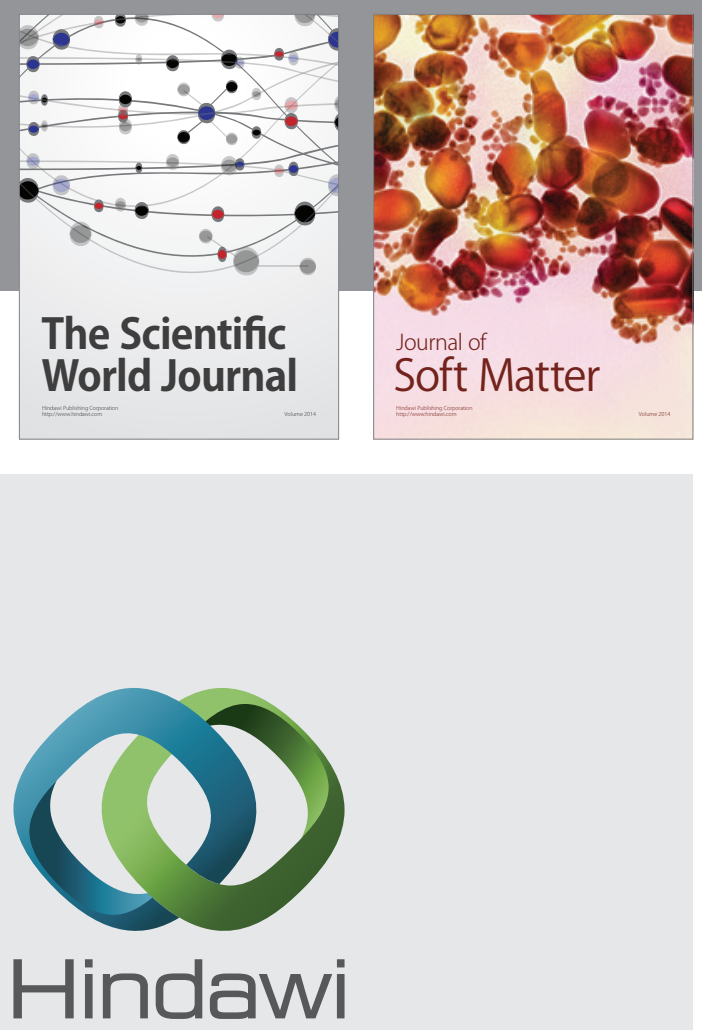

Submit your manuscripts at

http://www.hindawi.com

nternational Journal of

Statistical Mechanics
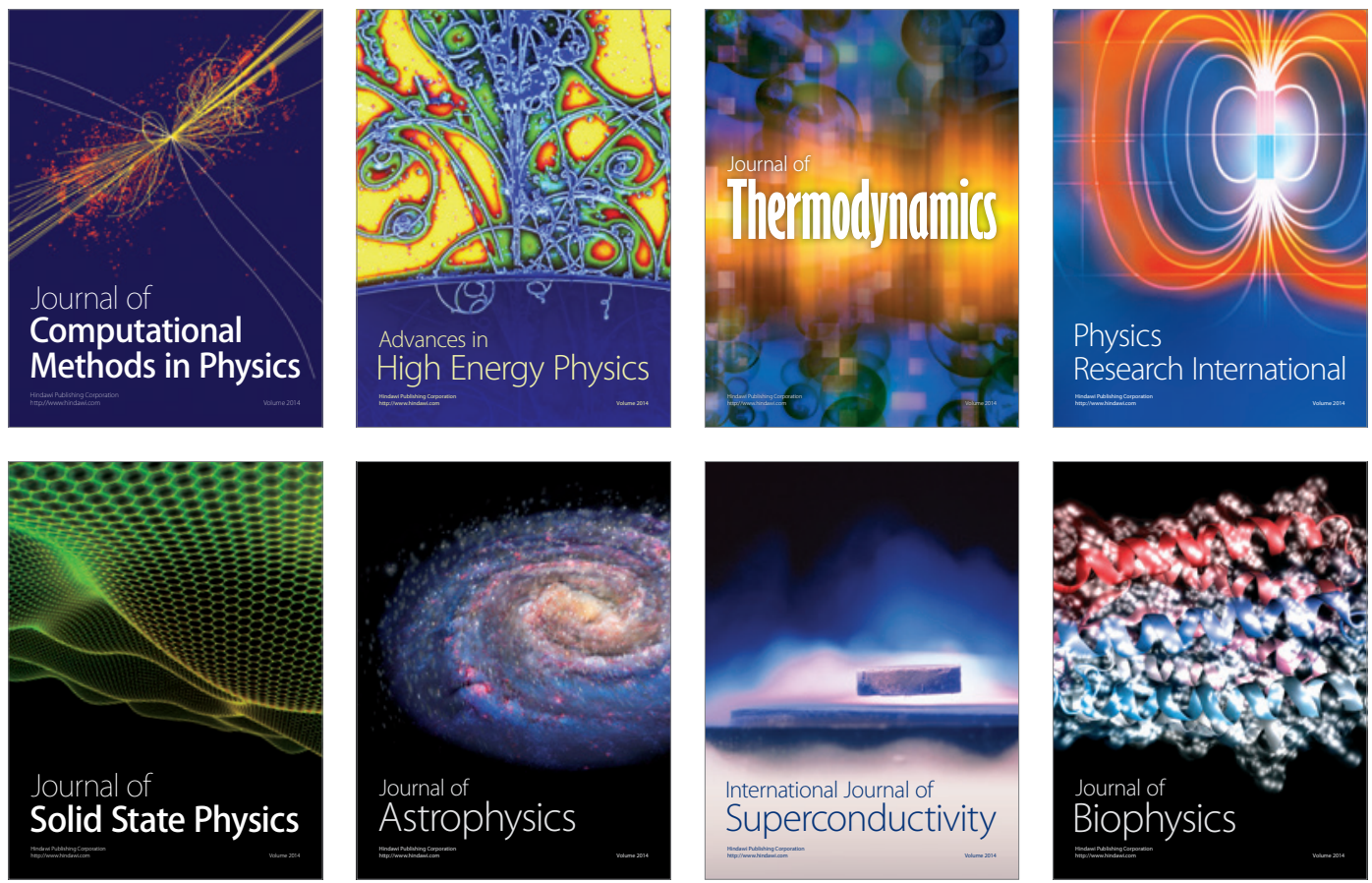
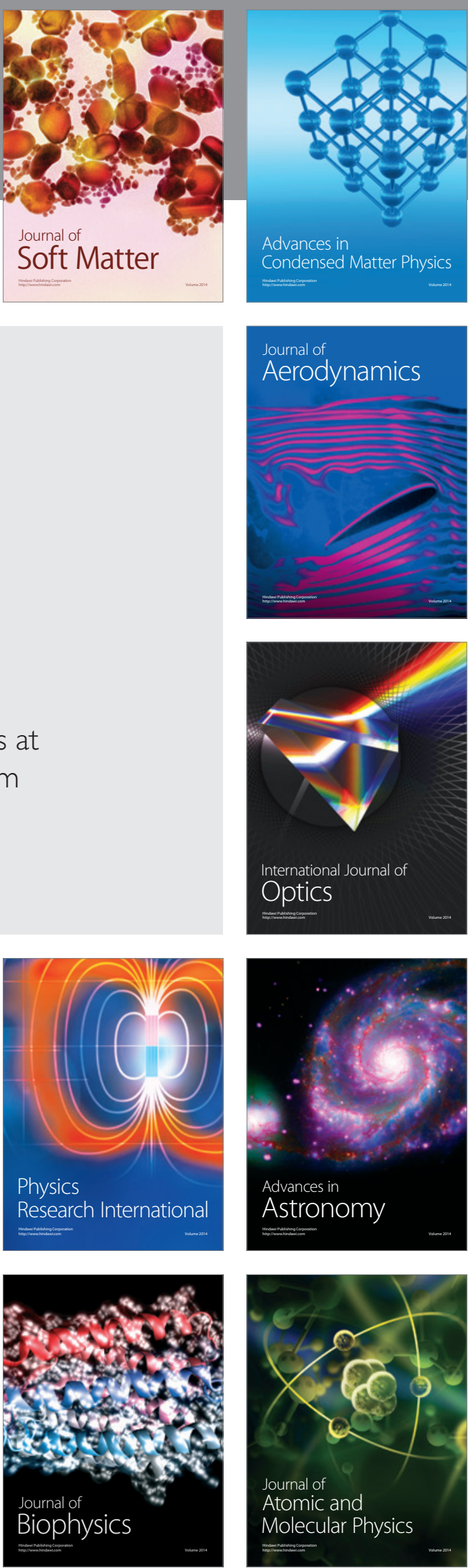